\title{
Brain MRI Depiction of Multiple Cranial Neuropathies in Neurosarcoidosis: A Case Report
}

\author{
Ken Ikedaa, b, Naoyuki Kawagoe ${ }^{\mathrm{a}}$, Takehisa Hirayama ${ }^{\mathrm{a}}$, Tetsuro Nagaoka ${ }^{\mathrm{a}}$, Konosuke Iwamoto ${ }^{\mathrm{a}}$, \\ Osamu Kano ${ }^{a}$, Kiyokazu Kawabe ${ }^{a}$, Yasuo Iwasaki ${ }^{a}$
}

\begin{abstract}
We report depiction of brain MRI lesions in a 33-year-old woman with multiple cranial neuropathies (II, III, V, VII, VIII, IX and X) due to neurosarcoidosis. She developed the right facial nerve palsy and vestibulopathy. MRI disclosed gadolinium-enhancement in the right geniculate ganglion and VIII cranial nerve. One week later, she had motor palsy of the left VII, IX and X cranial nerves, and hypoesthesia in the left $\mathrm{V}_{2}$ and $\mathrm{V}_{3}$ regions. Gadolinium-enhancing lesions were found in the bilateral IX and $\mathrm{X}$ cranial nerves, the pontine leptomeninges and the fourth ventricle. These neuroradiological hallmarks displayed that widespread basal meningitis and inflammation of the cranial nerves could contribute to the crucial pathogenesis of multiple cranial neuropathies in neurosarcoidosis.
\end{abstract}

Keywords: Neurosarcoidosis; Multiple cranial neuropathy; Basal meningitis; MRI; Gadolinium enhancement

\section{Introduction}

Sarcoidosis is an idiopathic granulomatous multisystem disease of unknown etiology. Cranial neuropathy is the common manifestation in neurosarcoidosis [1, 2]. Multiple cranial neuropathies involving 4 or more kinds of the cranial nerves were reported in a few patients with neurosarcoidosis [3, 4]. However, neuroradiological depiction remains unknown in those patients. We first highlight clinical and magnetic resonance imaging (MRI) findings in a patient with neurosar-

Manuscript accepted for publication August 19, 2011

${ }^{a}$ Department of Neurology, Toho University Omori Medical Center, 6 - 11 - 1, Omorinishi, Otaku, Tokyo, 143 - 8541, Japan

bCorresponding author: Ken Ikeda. Email: keni@med.toho-u.ac.jp

doi:10.4021/jnr47w coidosis who had basal meningitis and multiple neuropathies involving the II, III, V, VII, VIII, IX and X cranial nerves.

\section{Case Report}

A 33-year-old woman developed the right facial paralysis and unbalanced gait after dry cough and fever persisted for one month. She was admitted to department of otorhinolaryngology in our hospital. Neuro-otological examination revealed the right peripheral facial nerve palsy and vestibular dysfunction. Caloric test disclosed the right semicircular canal palsy. Brain MRI displayed gadolinium enhancement in the right VII and VIII cranial nerves (Fig. 1). She was diagnosed as Bell's palsy and received tapering intravenous administration of dexamethasone (10 mg/day) for 7 days. The right facial palsy and vestibular deficit were ameliorated markedly. After cessation of steroid therapy, she complained visual disturbance in the left eye. Subsequently diplopia, the left facial paralysis and hypoesthesia, dysphagia and dysphonia occurred. She came to our department for neurological consultation. Visual acuity was 20/16 in OD and 2/200 in

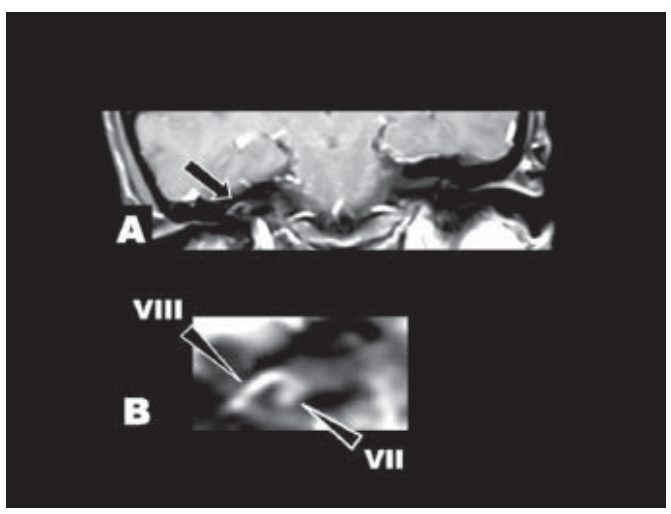

Figure 1. Post-gadolinium T1-weighted imaging at onset of the right VII and VIII cranial neuropathies. (A) Coronal view of the internal auditory canals. Abnormal enhancement was found in the right geniculate ganglion (VII) and the right VIII cranial nerve (arrow). (B) Enlarged view of the right internal auditory canal. Arrowheads indicated VII (the facial nerve) and VIII (the vestibular nerve). 


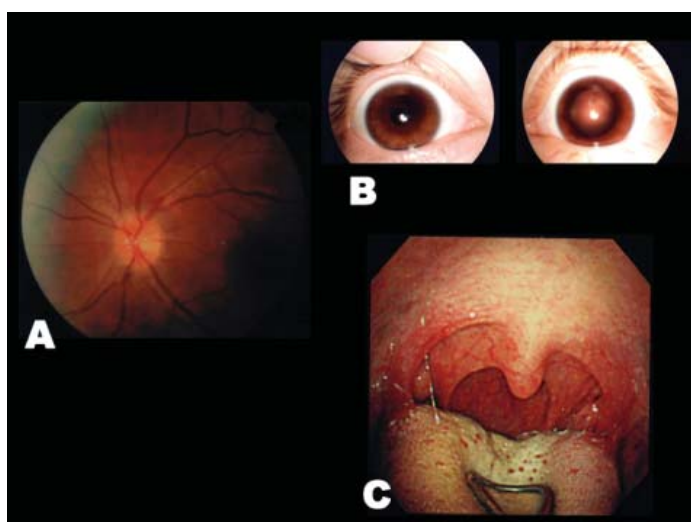

Figure 2. (A) Ophthalmoscopy showed edema in left optic discs. (B) Severe degree of the left mydriasis. (C) Laryngoscopy showed difficulty in elevating the left pharyngeal wall.

OS. Ophthalmoscopy showed edema of the left optic disc (Fig. 2A). Pupil size was $2.5 \mathrm{~mm}$ in the right side and 7.5 $\mathrm{mm}$ in the left side (Fig. 2B). Light reflex was absent in the left eye. Mild degree of the left superior rectus palsy was found on ocular movement. Neurological examination showed motor palsy of the left VII, IX and X cranial nerves, and hypoesthesia in the left $\mathrm{V}_{2}$ and $\mathrm{V}_{3}$ region. Laryngoscopy exhibited the left palatoparesis (Fig. 2C).

Serum levels of angiotensin-converting enzyme were increased to $28.9 \mathrm{U} / \mathrm{L}$ (normal ranges: 8.3 - 21.4). Cerebrospinal fluid study showed cell count of 55 mononuclear cell $/ \mathrm{mm}^{3}$, protein levels of $86 \mathrm{mg} / \mathrm{dL}$, cytology of class II and negative results of infectious pathogenic tests, includ- ing herpes simplex, tuberculosis, other bacteria and fungus. Myelin basic protein and oligoclonal Ig $\mathrm{G}$ bands were not detected. Chest X ray and computed tomography disclosed lymphadenopathy in the left pulmonary hilus and the right mediastinum. The CD4/CD8 ratio of bronchoalveolar lavage was elevated to 5.32 (normal < 3.4). Gallium scintigraphy of whole body revealed abnormal accumulation in the pulmonary hilus, the mediastinum and the parotid glands. Brain MRI displayed abnormal enhancement in the IX and the X cranial nerves, and the leptomeninges around the pons and the fourth ventricle (Fig. 3). She was diagnosed as basal meningitis and multiple cranial neuropathies due to neurosarcoidosis. Methylprednisolone pulse therapy (1,000 mg/ day for 3 days, iv) and the subsequent tapering administration of prednisolone (50 mg/day, po) were performed. Steroid treatment dramatically improved the left visual impairment, the left facial palsy and difficulty in the swallowing and the phonation. Afterwards, she had only mild degree of the left mydriasis.

\section{Discussion}

We reported clinicoradiological aspects in a distinct patient with neurosarcoidosis and multiple cranial neuropathies. The cranial nerve palsy occurred in the right VII and VIII, followed by the left II, III, V, VII, IX and X cranial nerves.

A previous study suggested that cranial neuropathy was presented in 35 of 54 patients with neurosarcoidosis (63\%) [2]. Of the cranial nerve involvement, the optic nerve was

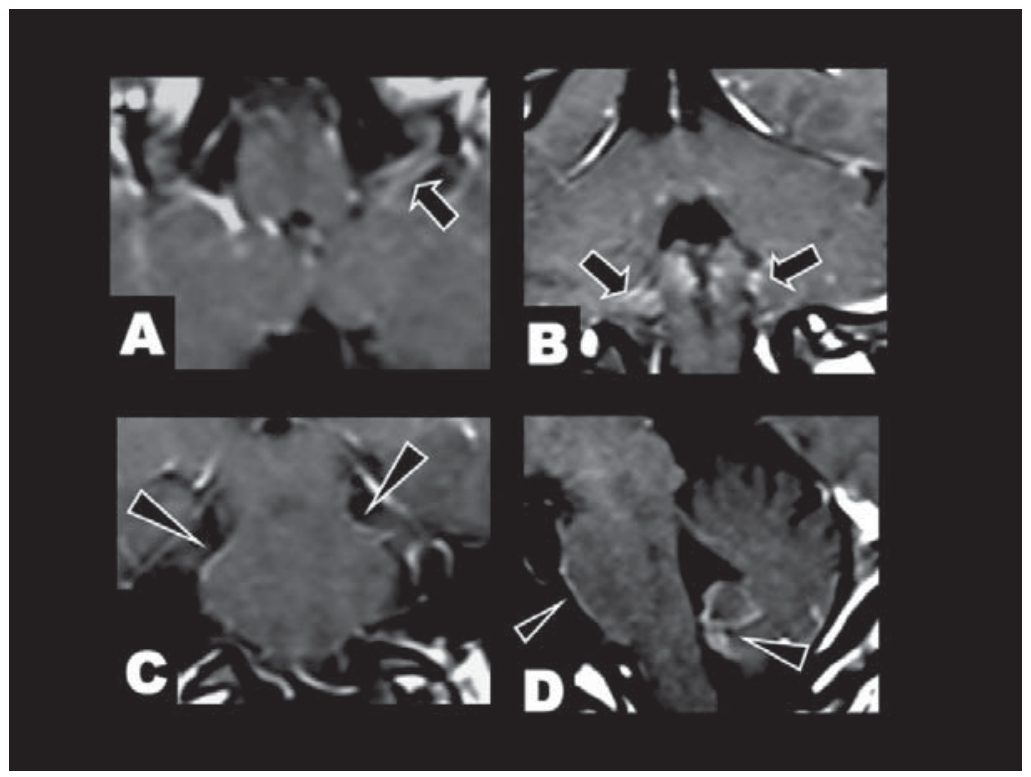

Figure 3. Post-gadolinium T1-weighted imaging at onset of the left IX and $X$ cranial neuropathies. (A) Axial and (B) coronal view. Abnormal enhancement was found in the bilateral IX and $X$ cranial nerves (arrows). (C) Coronal and (D) sagittal view. Leptomeningeal enhancement was found in the pons and the fourth ventricle (arrowheads). 
the most frequent lesion (35-38\%) and the facial nerve was the second most common (11-19\%) [1, 2]. Otherwise, 4 or more kinds of cranial neuropathies were extremely rare in neurosarcoidosis [3, 4]. The optic nerve is damaged by optic neuritis, intracranial hypertension or mass effect of intraorbital granuloma. The pathophysiological mechanism of the facial palsy is implicated in granulomatous mass, chronic meningitic reaction or secondary inflammation in the parotid gland. Previous MRI studies suggested variable changes in patients with neurosarcoidosis [1-3]. With respect to cranial nerve palsy, Oki et al first described a case that MRI showed enhanced mass in the distal internal auditory canal portion of the VII cranial nerve [5]. Thin-sliced (slice thickness $<3$ $\mathrm{mm}$ ) post-gadolinium T1-weighted imaging was a valuable tool for detecting the facial nerve lesion. In previous reports with multiple neuropathies of the II, III, VII, IX and X cranial nerves, brain MRI was normal. However, it was not mentioned whether gadolinium-enhanced MRI was performed in those cases [3, 4]. Gadolinium-enhanced brain MRI seems to display granulomatous massive lesions or chronic meningeal inflammation in patients with neurosarcoidosis. Otherwise, in many patients with cranial neuropathy, inflammatory lesions of the nerve tissue may be too small to detect on gadolinium-enhanced MRI, using conventional slice thickness. Brain MRI profile of our patient indicated abnormal enhancement in the non-massive lesions involving the VII, VIII, IX and X cranial nerves. Thus, these neuroimages supported that cranial nerve-targeted post-gadolinium MRI could succeed in depicting the inflammatory and small granulomatous changes in neurosarcoidosis patients with cranial neuropathy, in addition to basal meningitis.

In conclusion, here, we highlight clinical findings and brain MRI pictures of basal meningitis and 7 kinds of cranial neuropathies in a patient with neurosarcoidosis. The present patient provided that the cranial nerve tissues might be damaged by the direct neuronal inflammation and the spread of basal meningitis. These inflammatory changes could play a crucial role in the pathogenesis of multiple cranial neuropathies.

\section{Conflict of Interest}

All authors report no conflicts of interest.

\section{References}

1. Zajicek JP, Scolding NJ, Foster O, Rovaris M, Evanson J, Moseley IF, Scadding JW, et al. Central nervous system sarcoidosis--diagnosis and management. QJM. 1999;92(2):103-117.

2. Pawate S, Moses H, Sriram S. Presentations and outcomes of neurosarcoidosis: a study of 54 cases. QJM. 2009;102(7):449-460.

3. Heuser K, Kerty E. Neuro-ophthalmological findings in sarcoidosis. Acta Ophthalmol Scand. 2004;82(6):723729.

4. Erer-Ozbek S, Bora I, Yurtogullar S. Acute multiple cranial neuropathy in a patient with neurosarcoidosis. Neurol Sci. 2010;31(6):865-866.

5. Oki M, Takizawa S, Ohnuki Y, Shinohara Y. MRI findings of VIIth cranial nerve involvement in sarcoidosis. Br J Radiol. 1997;70(836):859-861. 